EFDA-JET-CP(05)02-18

A. Pospieszczyk, S. Brezinsek, A. Meigs, G. Sergienko, M. Stamp and JET EFDA contributors

\title{
Molecular (H/D/T) Sources in JET
}





\title{
Molecular (H/D/T) Sources in JET
}

\author{
A. Pospieszczyk ${ }^{2}$, S. Brezinsek ${ }^{2}$, A. Meigs ${ }^{1}$, G. Sergienko ${ }^{2}$, M. Stamp \\ and JET EFDA contributors*
}

\footnotetext{
${ }^{1}$ URATOM/UKAEA Fusion Association, Culham Science Centre, Abingdon, OX14 3DB, UK ${ }^{2}$ Institut für Plasmaphysik, Forschungszentrum Jülich GmbH, EURATOM Association, Trilateral Euregio Cluster, D-52425 Jülich, Germany

* See annex of J. Pamela et al, "Overview of JET Results ", (Proc. $2{ }^{\text {th }}$ IAEA Fusion Energy Conference, Vilamoura, Portugal (2004).
}

Preprint of Paper to be submitted for publication in Proceedings of the EPS Conference,

(Tarragona, Spain 27th June - 1st July 2005) 
"This document is intended for publication in the open literature. It is made available on the understanding that it may not be further circulated and extracts or references may not be published prior to publication of the original when applicable, or without the consent of the Publications Officer, EFDA, Culham Science Centre, Abingdon, Oxon, OX14 3DB, UK."

"Enquiries about Copyright and reproduction should be addressed to the Publications Officer, EFDA, Culham Science Centre, Abingdon, Oxon, OX14 3DB, UK." 


\section{INTRODUCTION}

Both tritium and deuterium will be used in equal measure as fuel species in future fusion reactors. A number of separate experiments have been conducted at JET over the years [1,2] in which tritium has been introduced in various quantities relative to the deuterium content. The most recent was the "trace tritium" campaign in 2003, in which tritium has been puffed both from the wall and injected via the neutral beam heating line [3]. Since gas puffed tritium is introduced as a molecule via the nozzle and recycles mainly in the form of hydrogen isotopomeres, it is of interest to investigate the formation, particularly of DT molecules, with regard to the total fuelling of T achieved in the discharge. This contribution describes the use of molecular spectroscopy, employed in a similar way to that reported in [5], to investigate DT and $\mathrm{T}_{2}$ molecule formation for the first time in JET. Tests with $\mathrm{H}_{2}$ injections have been carried out beforehand and will also be described in this paper. This method provides a very sensitive way to detect a low minority (hydrogen or tritium) concentration versus a high deuterium background level. Simultaneously it offers a unique possibility to record and study molecular spectra of both tritium and tritiated hydrogen isotopes in a fusion boundary plasma environment, which had never been performed in the past. In addition the full potential of molecular hydrogen spectroscopy developed for the determination of the molecular fluxes on TEXTOR [4] and on JET [5] could be employed

\section{EXPERIMENT AND SPECTROSCOPIC BACKGROUND}

In these experiments tritium has been introduced into the JET tokamak by short, $80 \mathrm{~ms}_{2}$ gas puffs of up to $5 \mathrm{mg}$ using a piezo-crystal valve (GIM 15) [6] located in the NB-injection line in octant 6. The quantity of injected tritium was maintained at very low levels (less than $1 \%$ of D) in order to keep the DT fusion rate low and decontamination time of the vessel short. The amount that is puffed is measured by the pressure drop in the reservoir of the valve and can be monitored by either Balmer spectroscopy of hydrogen isotope atoms or deuterium and tritium molecules. In [7] it was demonstrated that a low hydrogen content in the presence of walls saturated with deuterium could be determined by spectroscopy of molecular HD. This is illustrated in detail in fig.1 (for TD). One can see that from the measurement of the heteronuclear isotope (DT) a much smaller fraction of the homonuclear $\left(\mathrm{T}_{2}\right)$ species can be derived. Before tritium puffs were applied in JET this method could be tested in the same way by injecting $\mathrm{H}_{2}$ through GIM 15. In [5] the discharge conditions and the observation geometry used for the detection of the hydrogen isotopomeres formed in the outer divertor is described in detail as well as the molecular flux determination from the observation of the molecular Fulcher-a band emission $\left(3 \mathrm{p}^{3} \Pi_{\mathrm{u}} \rightarrow 62 \mathrm{~s}^{3} \Sigma_{\mathrm{g}}\right)$ in the relatively unperturbed red spectral wavelength range around $610 \mathrm{~nm}$.

\section{RESULTS}

Figure 2 displays the results of the hydrogen puff experiments. Four identical puffs at 17, 19, 22 and $24 \mathrm{~s}$ of $50 \mathrm{mbar} l$ for $100 \mathrm{~ms}$ each (green traces: $\mathrm{H}_{\alpha}$ ) were injected through GIM 15 into the L-mode 
phase of Pulse No: 61021 leading to a global density increase of about 6\% (fig.2(a)). The hydrogen to deuterium ratio at the location of injection was monitored by spectroscopy of $\mathrm{D}_{\alpha}$ and $\mathrm{H}_{\alpha}$ and amounted to about 7 (fig.2(b)). In octant 8 the simultaneous release of HD in the outer divertor could be observed in the Fulcher spectrum for the same times (fig.2(c)). As representative the Q3 line of the 0-0 transition was chosen, which is indicated in the extended reduced spectrum (fig.2(d)). It should be mentioned that the heteronuclear HD molecule displays no intensity alternation between odd and even Q-lines. (see [7]). From fig.2(d) one can deduce that the number of HD molecules amounts to about $10 \%$ of the $\mathrm{D}_{2}$ molecules which then results in a $0.2 \%$ contribution of $\mathrm{H}_{2}$ to the $\mathrm{D}_{2}$ flux (see fig.1). Similarly we find that the $\mathrm{H}$-atoms from molecules represent in this case $5 \%$ of the total molecular influx.

Figure 3 displays the results of the tritium puff experiments. In the L-mode phase of JET Pulse No:61384 one tritium pulse of $25 \mathrm{mbarl}$ for $80 \mathrm{~ms}$ was injected (green trace: Q3 from 0-0, see fig.3(b)). Simultaneously a global density increase of about $6 \%$ was observed, however, it is not clear if this is solely by the puff or a short switch of the discharge into an H-mode (fig.3(a)). The Fulcher- $\alpha$ spectrum of $\mathrm{T}_{2}$ can been clearly identified at the location of the gas puff (fig3(b)). This tritium data are the first obtained under fusion edge plasma conditions and complement now laboratory molecular

$\mathrm{T}_{2}$ spectra published more than 50 years ago produced under the very different discharge conditions of a discharge tube [8]. The tritium to deuterium ratio was found to be only two in this case, which might partly explain the lower TD level detected (as shown below).

Again it was tried to detect the simultaneous release of TD in the outer divertor in the Fulcher spectrum in octant 8 for the same time (fig.3(c)). It is difficult to find spectral ranges which display isolated TD lines without a higher resolving power. The reason for this is that with higher masses the levels and, hence, the transition ranges shrink, which make for small intensities blends from $\mathrm{D}_{2}$ bands more likely. The Q2 line of the 0-0 transition seems to be a more isolated one and is indicated in the extended reduced spectrum. The whole Q-branch of the 0-0 transition is only marginally visible and should again obey no intensity alteration similar to HD (fig.3(d)). In fig.3(d) one can estimate from Q2 that the number of TD molecules amounts to about $3 \%$ of the $\mathrm{D}_{2}$ molecules resulting in a $0.02 \%$ contribution of $\mathrm{T}_{2}$ to the $\mathrm{D}_{2}$ flux (see fig.1). The T-atoms from molecules represent in this case $1.5 \%$ of the total molecular influx, i.e. in both cases the $\mathrm{H} / \mathrm{T}$ in the heteronuclear molecules carry practically all the minority influx. A similar, but not so sensitive approach has been performed in [9] using tritiated hydrocarbon radicals.

\section{CONCLUSIONS}

Molecular hydrogen spectroscopy has proven to be a sensitive mean to detect very low amounts of the recycling flux without difficult deconvolution procedures necessary in the case of atomic species in magnetic fields. Moreover, the origin of the particle sources and the nature of particle release can be studied in detail via the ro-vibrational level population. 


\section{REFERENCES}

[1]. JET Team, Nucl. Fusion 32 (1992) 187

[2]. A. Gibson and the JET team, Physics of Plasmas 5 (1998) 1839

[3]. K-D. Zastrow, J. M. Adams, Yu. Baranov et al., PPCF 46 (2004) B255-B265

[4]. S. Brezinsek, G. Sergienko, A. Pospieszczyk, Ph. Mertens, U. Samm and P.T. Greenland, Plasma Phys. Control. Fusion 47 (2005) 615-634

[5]. A. Pospieszczyk, S. Brezinsek, G. Sergienko, P.T. Greenland, A. Huber, A. Meigs, Ph. Mertens, U. Samm, M. Stamp, S. Wiesen, J Nucl. Mater. 337-339 (2005) 500

[6]. T.T.C. Jones, Technical and scientific aspects of the JET trace-tritium experimental campaign 7th Tritium Science and Technology (Baden-Baden, Germany) 2004

[7]. S. Brezinsek, P. T. Greenland, Ph. Mertens, A. Pospieszczyk, U. Samm, B. Schweer G. Sergienko, Physica Scripta, T103 (2003) 63

[8]. G.H. Dieke and F.S. Tomkins, Phys.Rev. 76 (1949) 283

[9]. G. Duxbury, M.G. O’Mullane, H.P. Summers et al., $31^{\text {st }}$ EPS CPP, London, 2004 ECA Vol.28G, P-5.175 (2004).

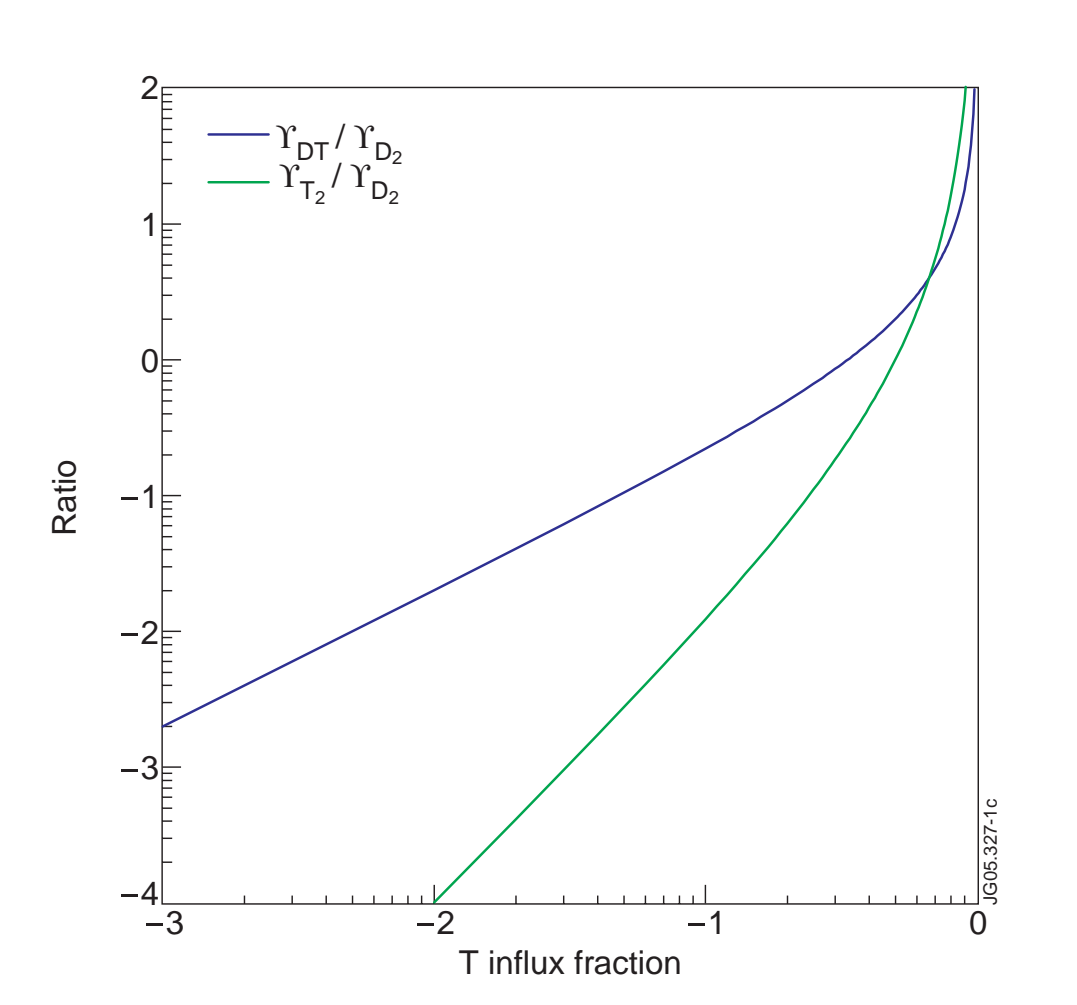

Figure 1: Molecular flux ratios versus surface concentration 


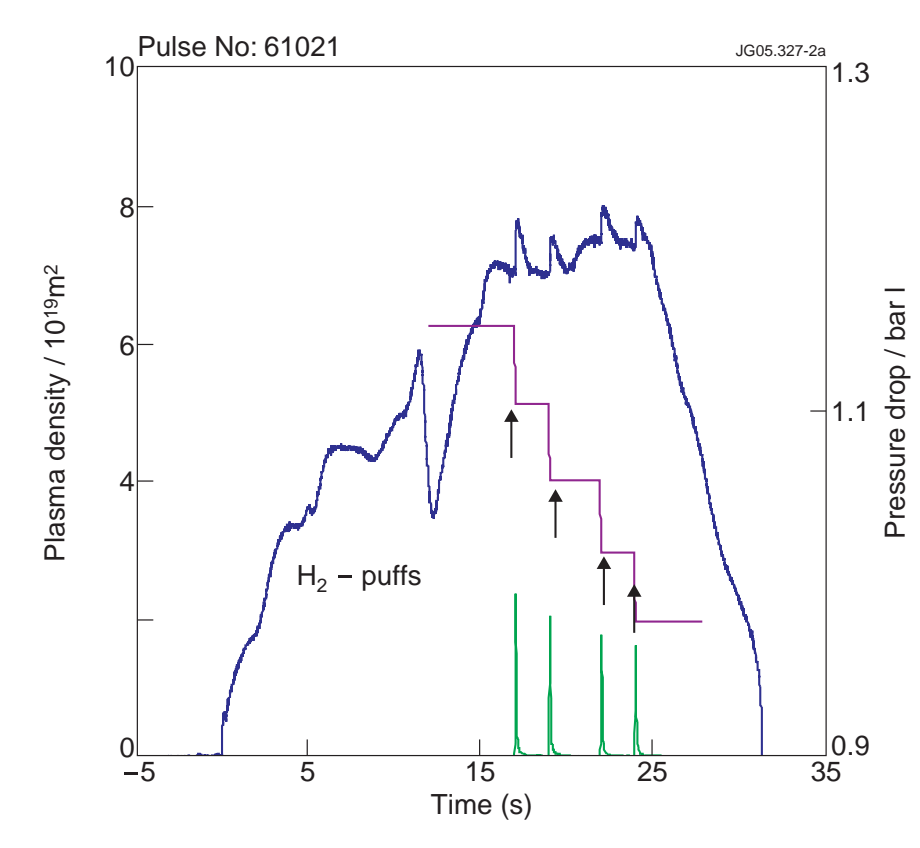

Figure 2(a): Hydrogen puffs into a D-plasma

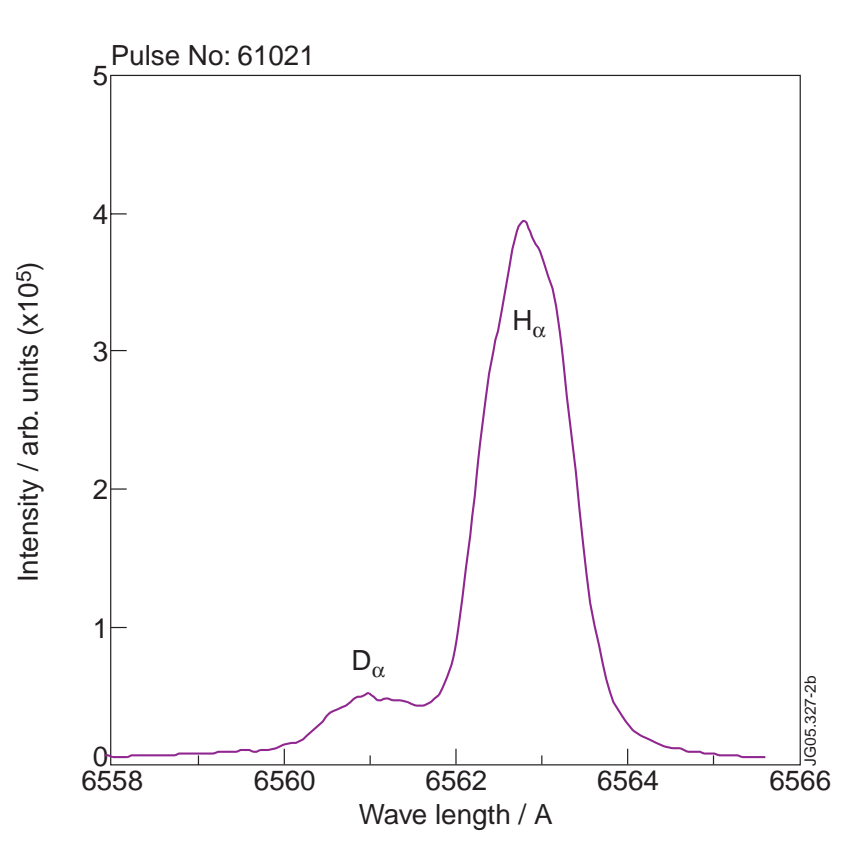

Figure 2(b): H / D Balmer- $\alpha$ lines near GIM 15

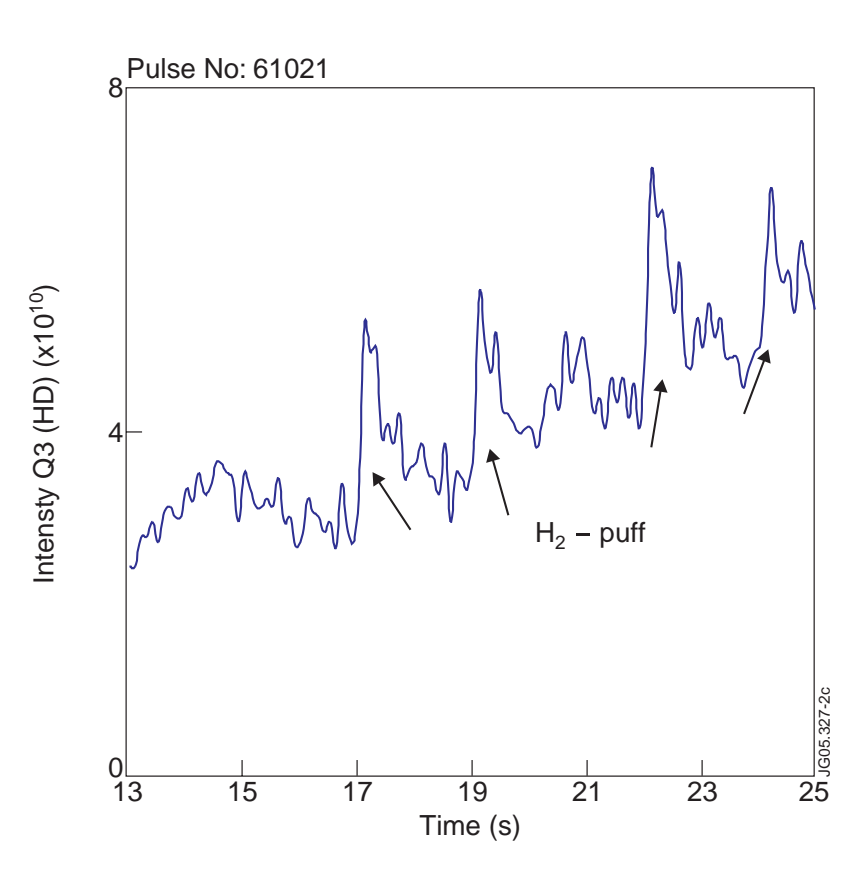

Figure 2(c): HD release versus time (outer divertor)

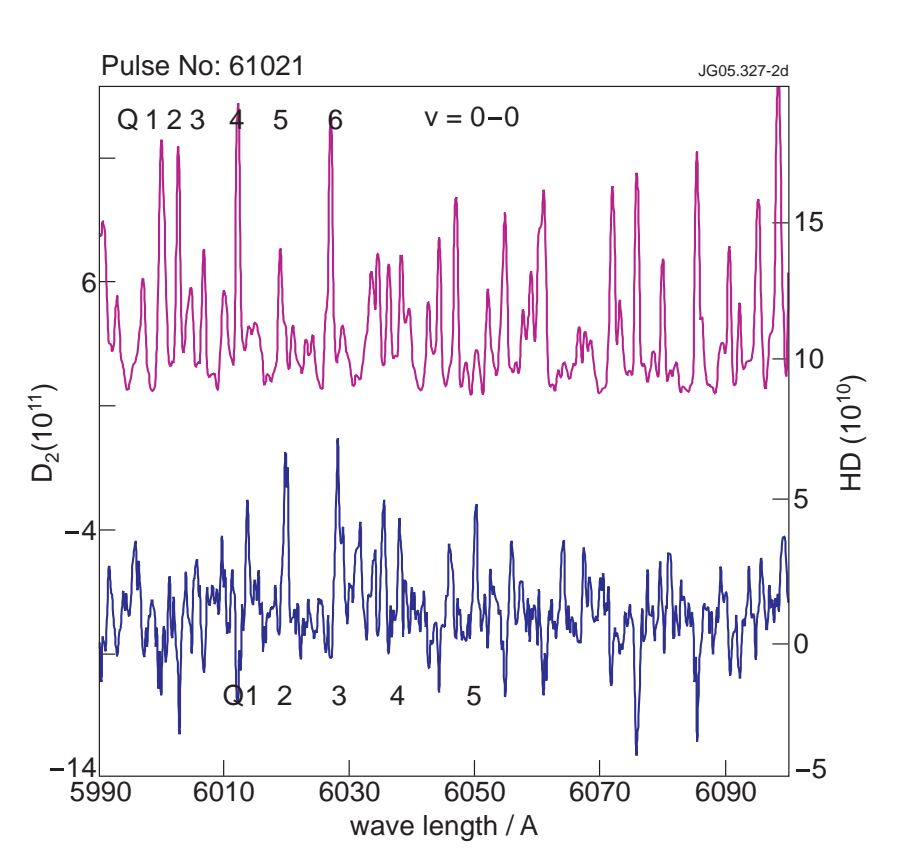

Figure 2(d): $D_{2}$ and $H D$ spectra before /during $H_{2}$ puff 


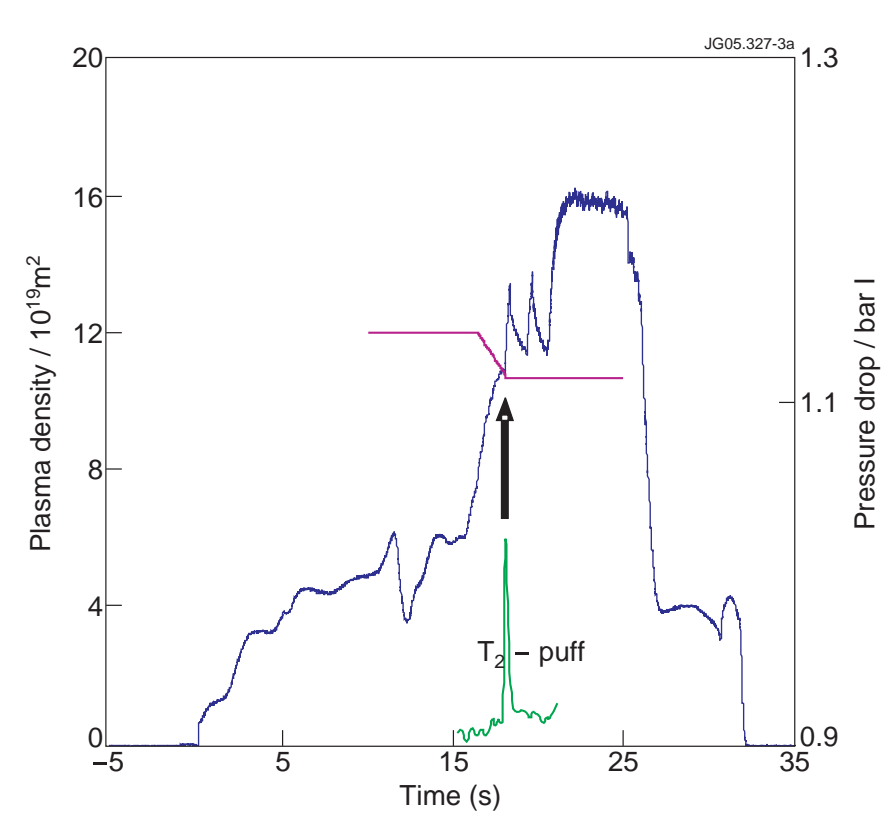

Figure 3(a): A tritium puff into a D-plasma

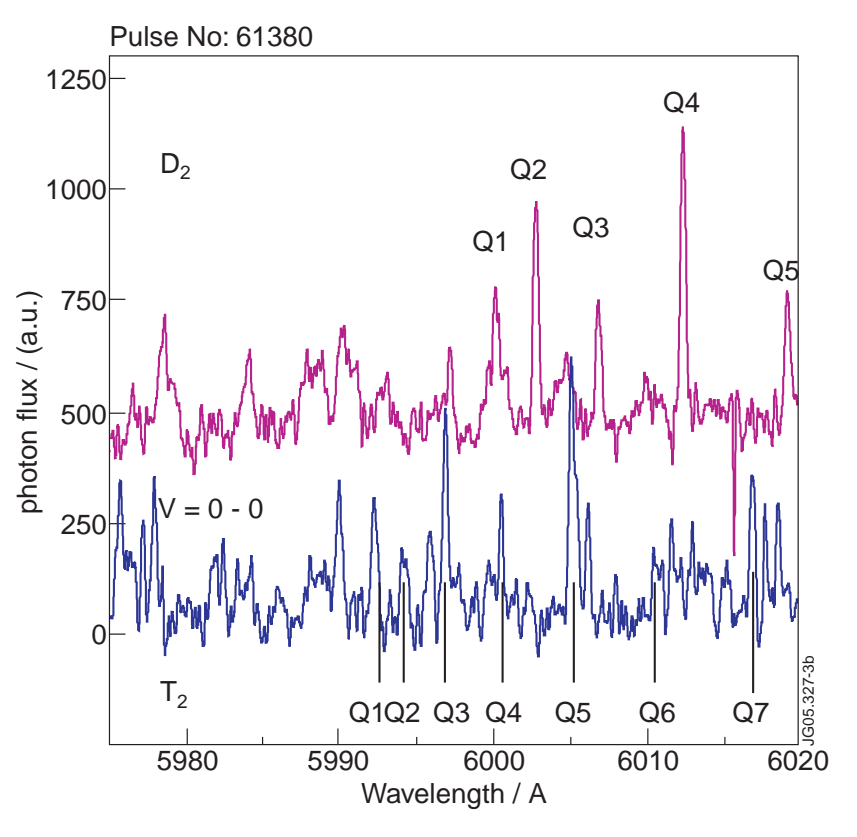

Figure 3(b): $D_{2}$ and $T_{2}$ spectra before / during the $T_{2}$ puff

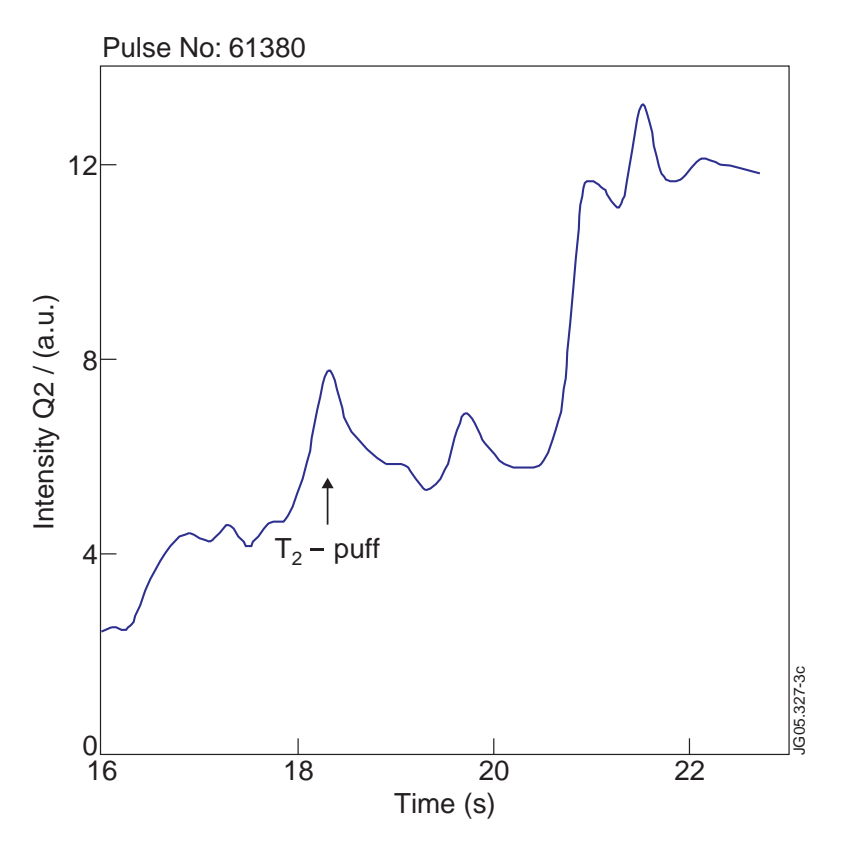

Figure 3(c): TD release versus time in the outer divertor

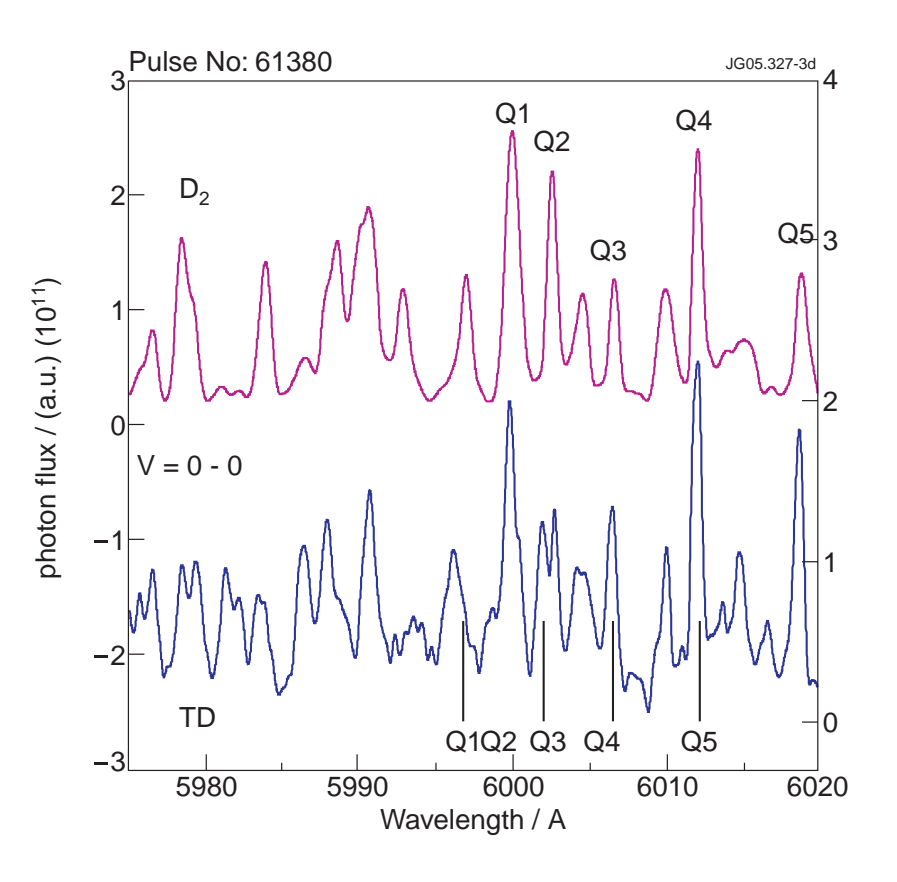

Figure 3(d): $D_{2} \&$ TD spectra before/during $T_{2}$ puff 